\title{
Structural basis for the recognition of K48-linked Ub chain by proteasomal receptor Rpn13
}

\author{
Zhu Liu ${ }^{1,2}$, Xu Dong ${ }^{1}$, Hua-Wei Yi ${ }^{1,3}$, Ju Yang ${ }^{1,3}$, Zhou Gong ${ }^{1}$, Yi Wang ${ }^{4}$, Kan Liu (1)', Wei-Ping Zhang $\mathbb{1}^{4}$ and \\ Chun Tang $\mathbb{B}^{1,3,5}$
}

\begin{abstract}
The interaction between K48-linked ubiquitin (Ub) chain and Rpn13 is important for proteasomal degradation of ubiquitinated substrate proteins. Only the complex structure between the N-terminal domain of Rpn13 (Rpn13 ${ }^{\mathrm{NTD}}$ ) and $\mathrm{Ub}$ monomer has been characterized, while it remains unclear how Rpn13 specifically recognizes K48-linked Ub chain. Using single-molecule FRET, here we show that K48-linked diubiquitin (K48-diUb) fluctuates among distinct conformational states, and a preexisting compact state is selectively enriched by Rpn $13^{\mathrm{NTD}}$. The same binding mode is observed for full-length Rpn13 and longer K48-linked Ub chain. Using solution NMR spectroscopy, we have determined the complex structure between Rpn $13^{\text {NTD }}$ and K48-diUb. In this structure, Rpn13 ${ }^{\text {NTD }}$ simultaneously interacts with proximal and distal Ub subunits of K48-diUb that remain associated in the complex, thus corroborating smFRET findings. The proximal Ub interacts with Rpn13 $3^{\mathrm{NTD}}$ similarly as the Ub monomer in the known Rpn13 $3^{\mathrm{NTD}}: \mathrm{Ub}$ structure, while the distal Ub binds to a largely electrostatic surface of Rpn $13^{\text {NTD }}$. Thus, a charge-reversal mutation in Rpn $13^{\text {NTD }}$ weakens the interaction between Rpn13 and K48-linked Ub chain, causing accumulation of ubiquitinated proteins. Moreover, physical blockage of the access of the distal Ub to Rpn13 ${ }^{\text {NTD }}$ with a proximity-attached Ub monomer can disrupt the interaction between Rpn13 and K48-diUb. Taken together, the bivalent interaction of K48linked Ub chain with Rpn13 provides the structural basis for Rpn13 linkage selectivity, which opens a new window for modulating proteasomal function.
\end{abstract}

\section{Introduction}

Ubiquitination is a post-translational modification, in which ubiquitin $(\mathrm{Ub})$, a 76-residue protein, is conjugated to a lysine residue of a substrate protein. The modifying $\mathrm{Ub}$ is referred to as proximal $\mathrm{Ub}$. Additional Ub units can

\footnotetext{
Correspondence: W.-P. Zhang (weiping601@zju.edu.cn) or

Chun Tang (tanglab@wipm.ac.cn)

${ }^{1}$ CAS Key Laboratory of Magnetic Resonance in Biological Systems, State Key Laboratory of Magnetic Resonance and Atomic Molecular Physics, National Center for Magnetic Resonance at Wuhan, Wuhan Institute of Physics and Mathematics of the Chinese Academy of Sciences, Wuhan, Hubei Province 430071, China

${ }^{2}$ National Key Laboratory of Crop Genetic Improvement, College of Life Science and Technology, Huazhong Agricultural University, Wuhan 430070, China

Full list of author information is available at the end of the article. These authors contributed equally: Zhu Liu, Xu Dong, Hua-Wei Yi
}

be further attached to the proximal $\mathrm{Ub}$, in which an isopeptide bond forms between the $\mathrm{C}$-terminal carboxylate group of the incoming $\mathrm{Ub}$ (referred to as distal $\mathrm{Ub}$ ) and an amine group of the proximal $\mathrm{Ub}$. The amine group in the proximal Ub can be one of the seven lysine side chains or $\mathrm{N}$-terminus, for a total of eight homotypic Ub linkages. Ub chains with different linkages encode different cell signals, among which K48-linked Ub chain can target the ubiquitinated substrate protein for proteasomal degradation.

The $26 \mathrm{~S}$ proteasome is a complex macromolecular machine that is made up of more than 30 types of proteins. Proteasomal degradation can be initiated upon specific interaction between substrate-conjugated K48linked Ub chain and ubiquitin receptor in the proteasome.

\section{(c) The Author(s) 2019}

(c) (i) Open Access This article is licensed under a Creative Commons Attribution 4.0 International License, which permits use, sharing, adaptation, distribution and reproduction cc) in any medium or format, as long as you give appropriate credit to the original author(s) and the source, provide a link to the Creative Commons license, and indicate if changes were made. The images or other third party material in this article are included in the article's Creative Commons license, unless indicated otherwise in a credit line to the material. If material is not included in the article's Creative Commons license and your intended use is not permitted by statutory regulation or exceeds the permitted use, you will need to obtain permission directly from the copyright holder. To view a copy of this license, visit http://creativecommons.org/licenses/by/4.0/. 
Three intrinsic Ub receptors have been identified, including Rpn $1^{1}, \operatorname{Rpn} 10 / \mathrm{S}^{2} \mathrm{a}^{2}$, and Rpn13/Adrm $1^{3}$, while the interactions between ubiquitin-like proteins in shuttle proteins and the proteasome as well as some other mechanisms may also be at play for targeting substrate proteins for degradation ${ }^{1,4,5}$. Structural characterizations of Rpn1, Rpn10, and Rpn13 have shown that, to achieve specific recognition, both proximal $\mathrm{Ub}$ and distal $\mathrm{Ub}$ in K48-linked diubiquitin (K48-diUb) interact with the two ubiquitin-interacting motifs in Rpn $10^{2}$, or with a toroid of helices in Rpn $1^{1}$. What is puzzling is that, only the complex structure between Ub monomer and Rpn13 Nterminal domain $\left(\operatorname{Rpn} 13^{\mathrm{NTD}}\right)$ has been determined ${ }^{6,7}$, and only a modest enhancement in binding affinity has been observed for Rpn13 interaction with K48-diUb than with Ub monomer ${ }^{3}$. As a result, it has been previously proposed that only the proximal Ub of K48-diUb interacts with Rpn13, while the distal Ub is free to interact with other proteins ${ }^{8,9}$. Thus, it remains to be established whether and how Rpn13 can interact with two Ub subunits simultaneously in the context of K48-linked Ub chain.

A $26 \mathrm{~S}$ proteasome comprises a $20 \mathrm{~S}$ core particle and two 19S regulatory particles stacked on both ends of the 20 S particle. Unlike Rpn1 and Rpn10, Rpn13 in the $19 \mathrm{~S}$ particle has not been visualized from the cryoEM images of human $26 \mathrm{~S}$ proteasome $\mathrm{e}^{10,11}$. There are several reasons for the missing electron density of Rpn13. First, biochemical and mass spectrometry analyses showed that only one copy of Rpn13 exists in the 26S proteasome, which means that Rpn13 is at 1:2 molar ratio with other regulatory proteins in the $19 \mathrm{~S}$ particles ${ }^{12}$. Second, Rpn13 is recruited to the proteasome by interacting with the C-terminal tail of $\mathrm{Rpn} 2^{7,13}$, which is largely unstructured $^{11,14}$. Therefore, Rpn13 is mobile with respect to the proteasome. Third, Rpn13 itself is dynamic, with a 150residue long flexible linker connecting Rpn $13^{\mathrm{NTD}}$ and the C-terminal domain.

Rpn13 plays redundant roles with Rpn1 and Rpn10 for Ub binding and substrate degradation. Yet, evidences have suggested that Rpn13 is uniquely involved in proteasomal degradation of certain proteins. Rpn13 is often overexpressed in malignant diseases ${ }^{15}$. Covalent binding of a small molecule to Rpn $13^{\mathrm{NTD}}$, occupying the binding site of Rpn2, can inhibit -ER stress-induced protein degradation and induce apoptosis ${ }^{16,17}$. As such, Rpn13 has been identified as an important target for the treatment of multiple myeloma and several other types of cancers. Rpn13 is also responsible for the degradation of IкB for the activation of NF-kB signaling pathway ${ }^{18}$. More interestingly, Rpn13 is subjected to ubiquitination at residue $\mathrm{K} 34^{19,20}$, and the modification attenuates proteasomal activity by perturbing the interaction between Rpn13 and K48-linked Ub chain ${ }^{21}$. Despite all the biological and pharmaceutical significances of Rpn13, it remains unclear how Rpn13 specifically recognizes K48-linked Ub chain to fulfill its function.

It has been shown that $\mathrm{Ub}$ can noncovalently interact with each other, and the noncovalent interactions are modulated by the covalent Ub linkage in di- or poly-Ub chain $^{22,23}$. The ligand-free K48-diUb has been crystalized in a fully closed state ${ }^{24}$. However, increasing evidences have indicated that K48-diUb also exists in open conformations in solution ${ }^{25-27}$. Furthermore, it has been shown that ligand-free K48-diUb fluctuates among multiple conformational states, ready to interact with different partner proteins ${ }^{26,27}$. While the open conformational states of K48-diUb are implicated in binding to K48linkage specific deubiquintase $\mathrm{e}^{26}$ and to E3-ligase ${ }^{28}$, the fully closed or compact state of K48-diUb is thought to represent a reservoir state ${ }^{25}$. This because in the compact state structure of ligand-free K48-diUb, key hydrophobic residues for interacting with other proteins, including L8, I44 and V70, are largely buried at the dimer interface ${ }^{24,27}$, and the two domains may have to open up for binding to specific partner proteins.

To understand how Rpn13 can be specifically recognized by K48-linked Ub chain, we resort to singlemolecule fluorescence resonance energy transfer $(\text { smFRET })^{29,30}$. The advantage of smFRET is that only two interacting molecules are investigated under near physiological conditions ${ }^{31}$. At the single-molecule level, complications from protein self-association and nonspecific interactions, commonly encountered in bulk measurements, can be minimized. Moreover, smFRET measurement allows rapid identification of constituting conformational states of protein structure, which would otherwise be difficult to deconvolute from the ensembleaveraged bulk measurement. In conjunction with smFRET measurements, we have used NMR spectroscopy to determine the solution structure of Rpn13 ${ }^{\mathrm{NTD}}: \mathrm{K} 48$ diUb complex. Together, we have shown that K48-diUb alternates among multiple conformational states, and it is the preexisting compact state of K48-linked Ub chain that selectively interacts with Rpn13.

\section{Results \\ K48-linked Ub chain dynamically fluctuates among multiple conformations}

We used smFRET to assess the spatial arrangement of the two Ub subunits in ligand-free K48-diUb. We introduced fluorophores, Alexa Fluor 488 and Cy5, at the Nterminus of the distal $\mathrm{Ub}$ and the C-terminus of the proximal Ub (Supplementary Fig. S1a). Using expectation maximization algorithm ${ }^{32}$, the smFRET profile of K48diUb can be best described as three overlapping FRET species (Supplementary Fig. S2). The high-, medium-, and low-FRET species are centered at FRET efficiencies of 

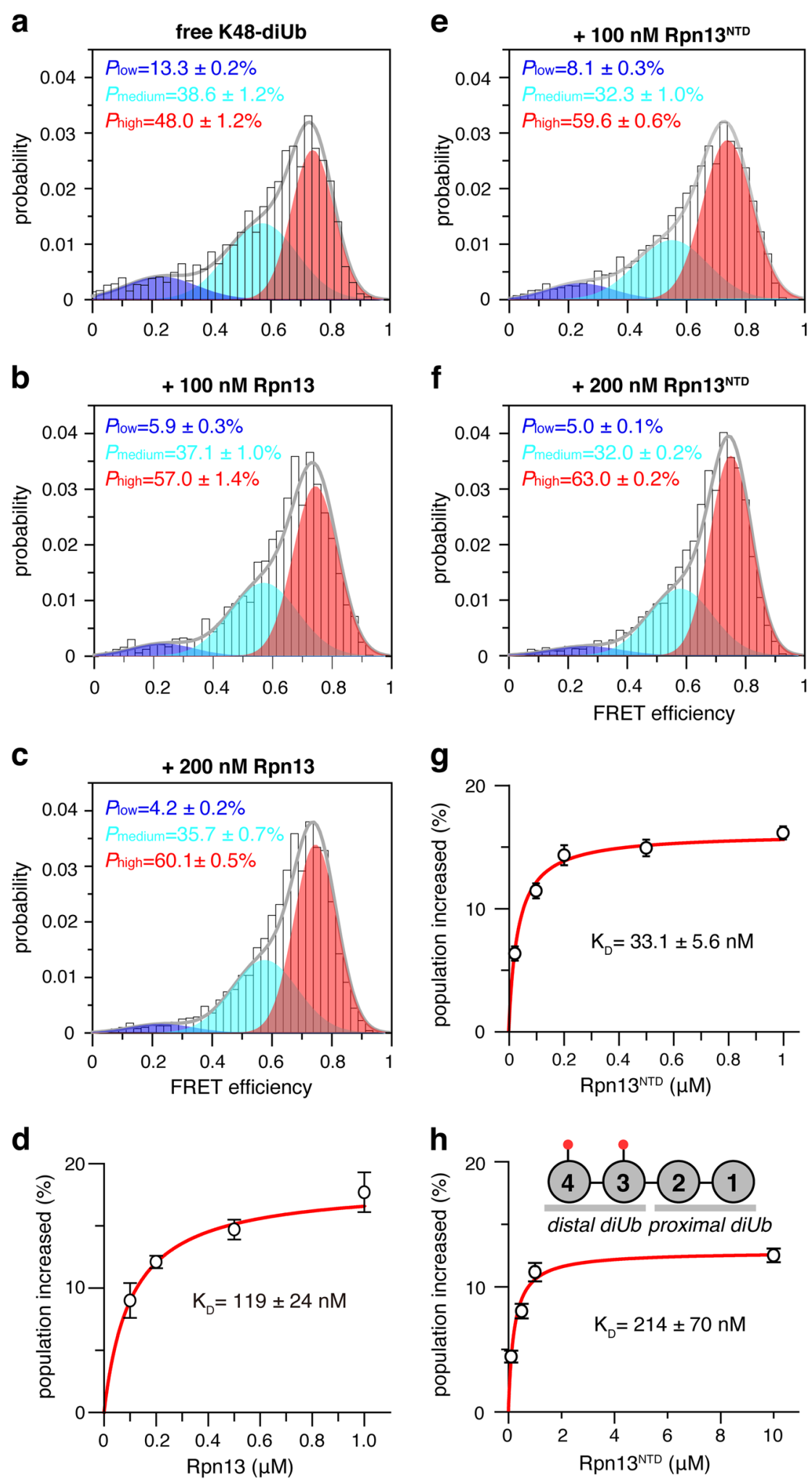

Fig. 1 (See legend on next page.) 
(see figure on previous page)

Fig. 1 Rpn13 recognizes a preexisting conformational state of K48-diUb. a With fluorophores conjugated at $76 \mathrm{C}$ site of the proximal Ub and 0 $\mathrm{C}$ of the distal Ub (cf. Supplementary Fig. S1a), the smFRET profile can be fitted to three overlapping FRET species. Unless otherwise noted, this pair of conjugation sites are used for all smFRET studies of K48-diUb. The high-FRET species (colored red) corresponds to a preexisting compact state. b-d The compact state can be selectively enriched by full-length Rpn13, and the population increase can be fitted to a binding isotherm. $\mathbf{e - g}$ The compact state can be selectively enriched by Rpn $13^{\mathrm{NTD}}$, and the population increase can be fitted to be a binding isotherm. $\mathbf{h}$ With the fluorophores conjugated at N25C/N25C sites, Rpn13 ${ }^{\text {NTD }}$ can selectively enrich the preexisting compact state of the distal diUb of K48-tetraUb (cf. Supplementary Fig. S5), affording a binding affinity. The populations of the smFRET species are averaged over three independent measurements, with the errors indicating $1 \mathrm{SD}$; the $\mathrm{K}_{\mathrm{D}}$ values are reported as best fit \pm fitting errors

$0.74,0.57$ and 0.23 , with respective populations of $\sim 48$, $\sim 39$, and $\sim 13 \%$ (Fig. 1a). The FRET distances between the center of fluorophores are calculated at $\sim 43, \sim 50, \sim 64 \AA$, respectively. Thus, the high-, medium-, and low-FRET species can be assigned to compact, semi-open, and open states that are preexisting for K48-diUb.

The conformational fluctuation of K48-diUb has been previously investigated using smFRET ${ }^{26}$. In that study, the authors resolved two smFRET species for K48-diUb, namely high-FRET and low-FRET species with center FRET efficiencies at 0.69 and 0.41 , respectively, in addition to a no-FRET species. The authors showed that the titration of an inactivated OTUB1 (OTUB1i), a deubiquitinase specific for $\mathrm{K} 48$ isopeptide linkage ${ }^{33}$, mainly enriches the low-FRET species. Their observation led to the proposal that K48-diUb can be specifically recognized by OTUB1 through a conformational selection mechanism. Here we repeated the smFRET titration, and found that OTUB1i enriches the medium-FRET species (Supplementary Fig. S3a-c). Moreover, the populational increase of the medium-FRET species upon OTUB1i titration can be fitted to a binding isotherm with a $K_{D}$ value of $7.7 \pm 0.1 \mu \mathrm{M}$ (Supplementary Fig. S3d), which is close to the $K_{D}$ value previously reported ${ }^{26}$. Thus, the medium-FRET species in the present study should correspond to the low-FRET species in the previous study, and the discrepancy may arise from different photon counting efficiencies and fitting routines of smFRET time traces.

To further confirm that K48-diUb fluctuates among three preexisting conformational states, we introduced fluorophores at additional pairs of fluorophore conjugation sites (Supplementary Fig. S1b-d). For the alternative sites, although the center efficiencies of the FRET species differ, the smFRET profiles can still be described as three overlapping FRET species with similar populations (Supplementary Fig. S4). For example, for the $25 \mathrm{C} / 25 \mathrm{C}$ conjugation sites, the high-, medium-, and low-FRET species are centered at FRET efficiencies of 0.68, 0.54 and 0.21 , with respective populations of $\sim 48 \%, \sim 43 \%$, and $\sim 9 \%$ (Supplementary Fig. S4d). Thus, conjugation of the fluorophores is unlikely to perturb protein structure, and the smFRET measurements have revealed the inherent conformational dynamics of K48-diUb regardless of the conjugation site, that is, the K48-diUb alternates among three distinct states in the absence of a partner protein.

To further assess whether Ub subunits in longer K48linked Ub chain also fluctuates among multiple conformational states, we analyzed the smFRET profile of K48-linked tetra-ubiquitin (K48-tetraUb). We conjugated the fluorophores at two $\mathrm{N} 25 \mathrm{C}$ sites in the distal diUb (with respected to the proximal diUb) of K48-tetraUb. The smFRET profile can also be fit as three overlapping FRET species (Supplementary Fig. S5a). Although the relative populations of the three species are different from that of an isolated K48-diUb with fluorophores conjugated at same sites (Supplementary Fig. S4d), the center FRET efficiencies of the FRET species are almost identical. Thus, the conformational states of the diUb unit is likely preserved in longer K48-linked Ub chain, while the difference in the relative populations can be a result of modulatory effect of the proximal diUb.

\section{The high-FRET species is selectively enriched by Rpn 13}

To assess the relationship between the conformational dynamics of K48-linked Ub chain and Rpn13 recognition, we titrated 150 pM fluorophore-labeled K48-diUb with human full-length Rpn13 protein. Interestingly, upon the addition of $100 \mathrm{nM}$ Rpn13, the preexisting high-FRET species of K48-diUb is enriched from $\sim 48 \%$ to $\sim 57 \%$ (Fig. 1a, b), while the population of medium- and lowFRET species decreases. The population of high-FRET species continues to increase with more Rpn13 added (Fig. 1c), and the binding isotherm can be fitted to a $K_{D}$ value of $119 \pm 24 \mathrm{nM}$ (Fig. 1d).

It has been previously shown that Rpn $13^{\mathrm{NTD}}$ is mainly responsible for Ub binding ${ }^{6,7}$. Thus, we performed smFRET titration for 150 pM fluorophore-labeled K48diUb using only Rpn $13^{\text {NTD }}$ comprising only the first 150 residues. Rpn $13^{\text {NTD }}$ also selectively enriches the highFRET species of K48-diUb (Fig. 1e, f). The population increase of high-FRET species can be fitted to afford a $K_{D}$ value of $33.1 \pm 6.9 \mathrm{nM}$ (Fig. 1g), which is about 4-fold increase in affinity as compared to full-length Rpn13. If the fluorophores are attached at alternative conjugation sites (Supplementary Fig. S1b and S4b), titration of 
Rpn13 ${ }^{\mathrm{NTD}}$ also causes the enrichment of equivalent FRET species following a similar trend, affording almost identical $K_{\mathrm{D}}$ value (Supplementary Fig. S6). Thus, the appearance additional residues may have a small inhibitory effect on the interaction between $\mathrm{Rpn} 13^{\mathrm{NTD}}$ and K48-diUb.

Furthermore, we performed smFRET titration for $150 \mathrm{pM}$ K48-tetraUb with fluorophores conjugated at the distal diUb (Supplementary Fig. S5a). Titration of $R p n 13^{\text {NTD }}$ selectively enriches the preexisting high-FRET species of the fluorophore-labeled distal diUb (Supplementary Fig. S5b-d), and the binding isotherm can be fitted to a $K_{\mathrm{D}}$ value of $214 \pm 70 \mathrm{nM}$ (Fig. 1h). The 7 -fold reduction in binding affinity compared to Rpn $13^{\mathrm{NTD}}: \mathrm{K} 48$ diUb interaction can be attributed to the self-association between distal diUb and proximal $\mathrm{diUb}^{34}$, making the binding surface less available for Rpn13 binding. Importantly, for all the smFRET titrations of K48-diUb or K48tetraUb, the center efficiency of the high-FRET species changes little in the absence or presence of Rpn13 or Rpn $13^{\text {NTD }}$. This means that Rpn13 binds to a preexisting conformation of K48-diUb through a conformational selection mechanism, whether K48-diUb is by itself or part of longer Ub chain. It also means that the high-FRET species, i.e. the preexisting compact state of K48-diUb, is not fully closed, and is ready to interact with other proteins. Importantly, the selective enrichment of the highFRET species also indicates that Rpn13 should interact with both Ub subunits at the same time.

\section{Rpn13 preferentially binds to K48-linked diUb}

To assess Rpn13 binding specificity for K48 linkage, we assessed the binding affinities between Rpn13 and other types of $\mathrm{Ub}$ proteins. With the titration of $200 \mathrm{nM}$ Rpn $13^{\text {NTD }}$ into $150 \mathrm{pM}$ fluorophore-labeled K48-diUb, the population of the high-FRET species increases by $15 \%$ from $\sim 48 \%$ to $\sim 63 \%$ (Fig. 1f). At this concentration, K48diUb binding is not yet saturated with Rpn13 ${ }^{\text {NTD }}$ (Fig. 1g) and therefore, the population of the high-FRET species is sensitive to small change of the available $R p n 13^{\text {NTD }}$ concentration. Unlabeled $\mathrm{K} 48-\mathrm{diUb}$ can compete for the binding to Rpn $13^{\mathrm{NTD}}$ with fluorophore-labeled K48-diUb. With the addition of $150 \mathrm{pM}$ unlabeled K48-diUb, the population of the high-FRET species decreases by 7.5\%, corresponding to a $50 \%$ inhibition of Rpn $13^{\mathrm{NTD}}$-bound fluorophore-labeled K48-diUb (Fig. 2a). With the addition of 300 pM unlabeled K48-diUb, the population of highFRET species decreases by $11 \%$, amounting to a total of 73\% inhibition (Fig. 2b). As such, both fluorophorelabeled and unlabeled K48-diUb compete for the same binding interface on Rpn13 with similar binding affinity. This also means that conjugation of the fluorophores to K48-diUb causes little perturbation to the interaction between Rpn13 ${ }^{\mathrm{NTD}}$ and K48-diUb.
Furthermore, to the mixture of $200 \mathrm{nM} \mathrm{Rpn} 13^{\mathrm{NTD}}$ and $150 \mathrm{pM}$ fluorophore-labeled K48-diUb, we added unlabeled Ub monomer, K63-linked diubiquitin or M1-linked diubiquitin, to assess whether other types of Ub can displace K48-diUb. The population of the high-FRET species change little with the addition of $150 \mathrm{pM}$ or $300 \mathrm{pM} \mathrm{Ub}$ monomer (Fig. 2c, d). With the addition of $150 \mathrm{pM}$ K63diUb and $150 \mathrm{pM}$ M1-diUb, the population of the highFRET species are also unchanged within the error range (Fig. 2e, f). On the other hand, direct titration of $1 \mu \mathrm{M}$ Rpn13 ${ }^{\text {NTD }}$ into fluorophore-conjugated K63-diUb and M1-diUb causes little change to their preexisting smFRET profiles (Supplementary Fig. S7). Together, Rpn13 selectively interacts with K48-diUb.

\section{Solution structure of Rpn13 ${ }^{\mathrm{NTD}}: \mathrm{K} 48$-diUb complex}

Though we have now shown Rpn $13^{\text {NTD }}$ selectively interacts with K48-diUb, only the complex structure between $\mathrm{Rpn} 13^{\mathrm{NTD}}$ and Ub monomer has been determined $^{6,8}$. To understand how the two subunits in K48diUb can simultaneously interact with $\operatorname{Rpn} 13^{\mathrm{NTD}}$, we set out to determine the solution structure of Rpn $13^{\mathrm{NTD}}: \mathrm{K} 48$ diUb complex using nuclear magnetic resonance (NMR). Upon the formation of protein complex, interfacial residues would experience different local environment and therefore display NMR signals. We found that, titrating unlabeled K48-diUb to ${ }^{15} \mathrm{~N}$-labeled Rpn13 causes large chemical shift perturbations (CSPs), which mainly involve residues 73-83 and 93-106 (Fig. 3a). These residues form a contiguous surface on Rpn13, covering an area larger than expected from the previously determined complex structure between Rpn $13^{\mathrm{NTD}}$ and Ub monomer ${ }^{6,7}$. On the other hand, titrating unlabeled Rpn $13^{\mathrm{NTD}}$ to K48-dUb, with either proximal or distal $\mathrm{Ub}{ }^{15} \mathrm{~N}$-labeled and the other subunit unlabeled, the CSPs are mainly observed for residues in the $\beta$-sheet region of both Ub subunits. Some of the interfacial residues also disappeared upon the formation of the complex (Fig. 3b, c).

Nuclear Overhauser effect (NOE) reports distance relationship $\left(<6 \AA\right.$ ) between nuclei. Moreover, ${ }^{13} \mathrm{C}$ halffiltered NMR experiment can provide NOE between ${ }^{12} \mathrm{C}$ bonded proton and ${ }^{13} \mathrm{C}$-bonded proton, i.e. intermolecular distance relationship. We obtained intermolecular NOEs between Rpn13 ${ }^{\text {NTD }}$ and the proximal $\mathrm{Ub}$, between Rpn13 ${ }^{\mathrm{NTD}}$ and the distal Ub, and between proximal Ub and distal Ub (Supplementary Fig. S8). Furthermore, we conjugated a maleimide-EDTA- $\mathrm{Mn}^{2+}$ paramagnetic probe at E24C site of the distal Ub, and collected paramagnetic relaxation enhancement (PRE) for backbone amide protons of Rpn $13^{\mathrm{NTD}}$, following the established protocol ${ }^{22,35}$. As assessed either by peak intensity ratio of paramagnetic versus diamagnetic spectra or by transverse relaxation enhancement $\Gamma_{2}$ rate, Rpn13 residues 30-42 and 101-106 experience large PREs with 

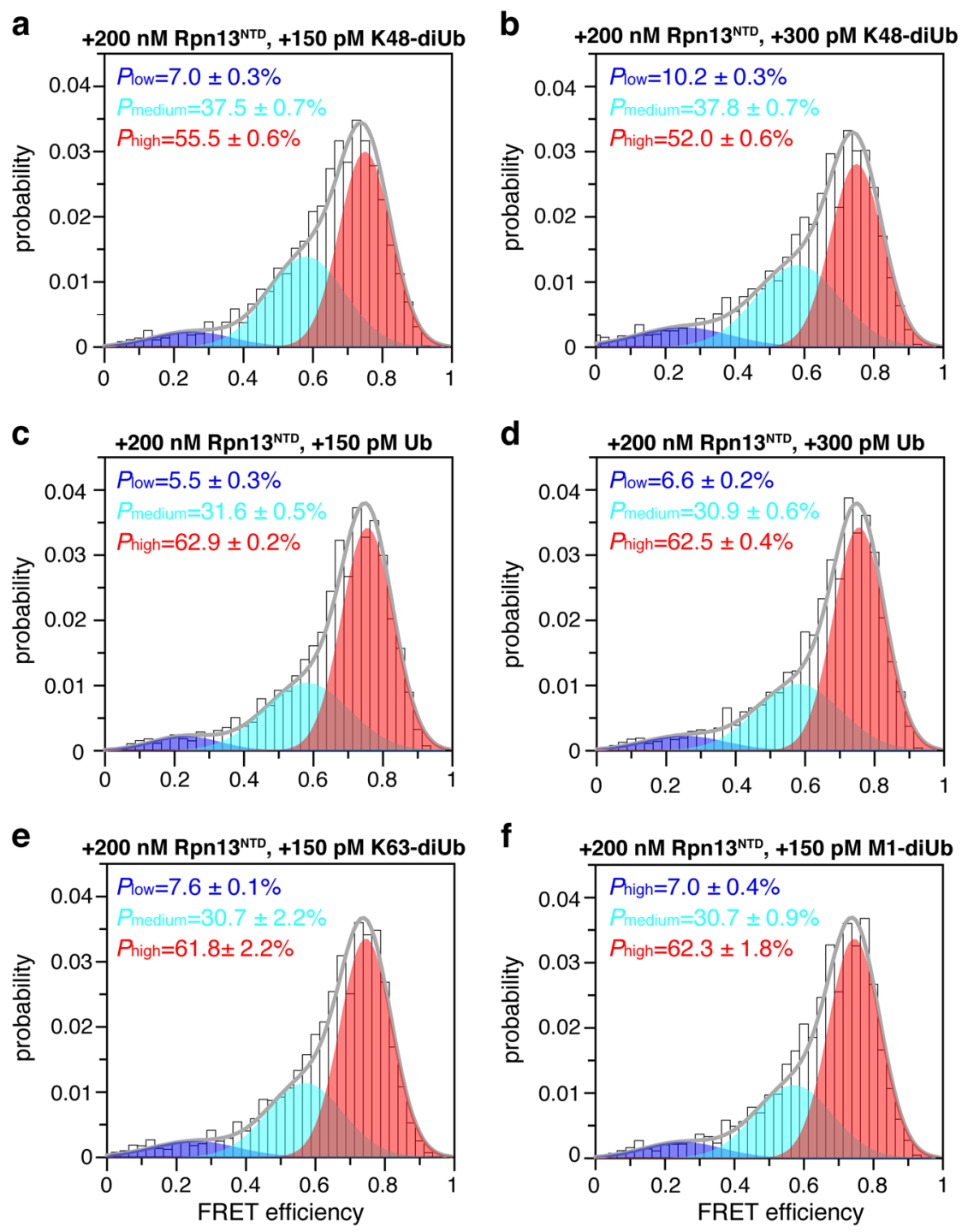

Fig. 2 Linkage selectivity of Rpn13. a, b Addition of $200 \mathrm{nM}$ Rpn13 $3^{\mathrm{NTD}}$ first enriches the high-FRET species of fluorophore-labeled K48-diUb (cf. Figure 1f), and further addition of $150 \mathrm{pM}$ and $300 \mathrm{pM}$ unlabeled K48-diUb decrease the population of the high-FRET species. c, d Addition of 150 pM and 300 pM unlabeled Ub monomer has negligible effect on the population of the high-FRET species. e, f Addition of 150 pM K48-diUb and 150 pM M1-diUb cause very small decrease of the population of the high-FRET species

severe line broadening (Fig. 3d, e). We also conjugated the paramagnetic probe at $\mathrm{N} 25 \mathrm{C}$ site of the proximal $\mathrm{Ub}$, and evaluated the transverse relaxation enhancement rate $\Gamma_{2}$ for the distal Ub (Fig. 3f). Large PRE values are observed between the two Ub subunits of the ligand-free K48-diUb, and the addition of Rpn $13^{\text {NTD }}$ increases inter-Ub PREs but with a similar PRE profile. The PRE NMR experiments thus confirm that Rpn $13^{\mathrm{NTD}}$ enriches the preexisting compact state of K48-diUb.

To refine the Rpn13 ${ }^{\mathrm{NTD}}: \mathrm{K} 48$-diUb complex structure against experimental restraints, we performed rigid body docking with torsion angle freedom given to the diUb linker residues and to the side chains of interfacial residues. For the 20 lowest-energy conformers, the rootmean-square (RMS) deviation for backbone heavy atoms of all rigid residues is $0.86 \pm 0.54 \AA$ (Supplementary Fig. S9 and Table S1). The two Ub subunits of K48-diUb remain associated in the complex, burying solvent accessible surface area (SASA) of $\sim 1130 \AA^{2}$. On the other hand, Rpn $13^{\text {NTD }}$ wedges in, burying $\sim 940 \AA^{2}$ of SASA with the proximal $\mathrm{Ub}$ and $\sim 1300 \AA^{2}$ of SASA with the distal Ub (Fig. 4a). The complex structure between Rpn $13^{\text {NTD }}$ and proximal Ub of K48-diUb in the present study is similar to the known complex structure between Rpn $13^{\text {NTD }}$ and Ub monomer ${ }^{6,7}$, with the RMS difference for backbone heavy atoms $2.17 \pm 0.31 \AA$ (Supplementary 

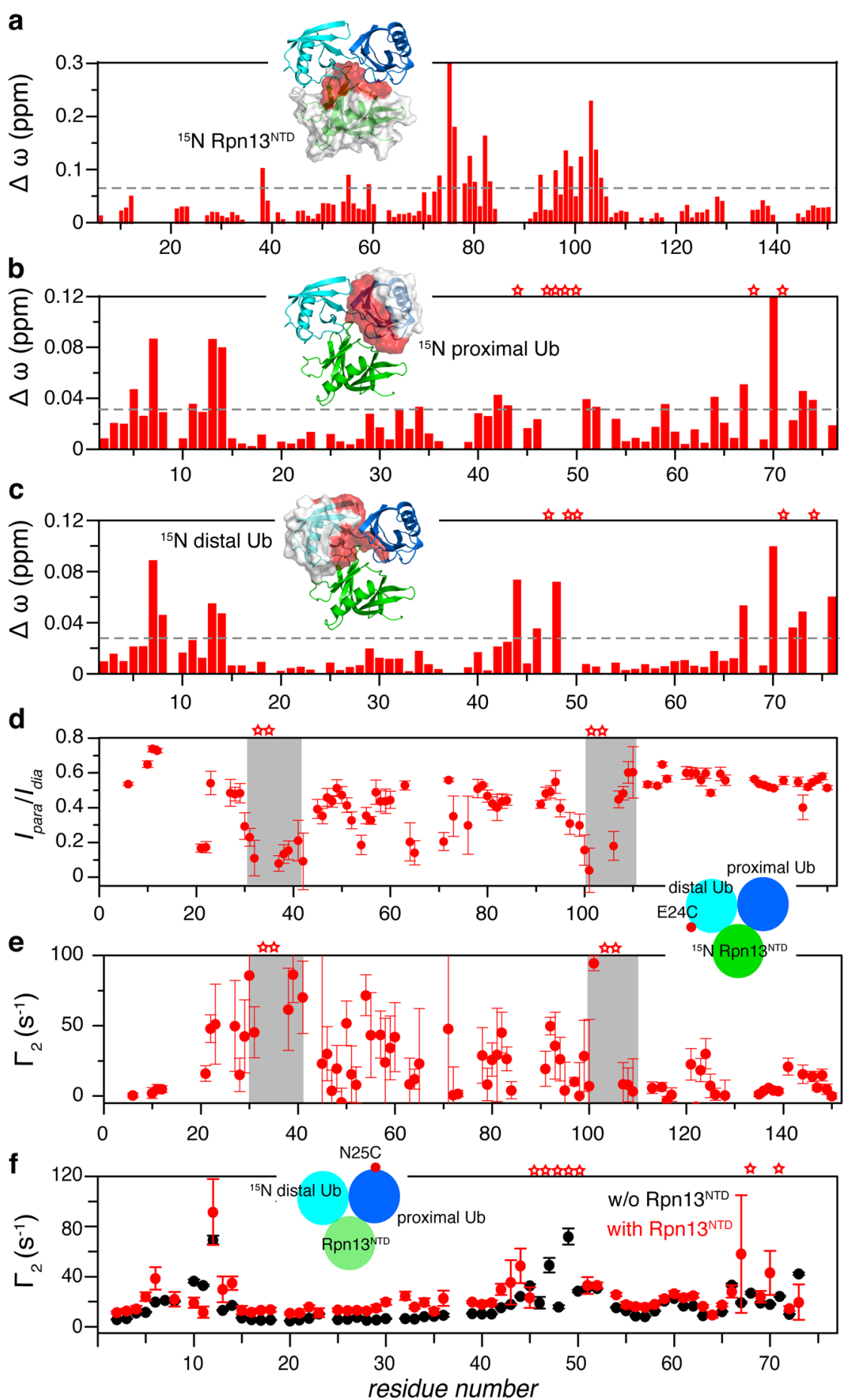

Fig. 3 Structural characterization of Rpn13 ${ }^{\mathrm{NTD}}$ :K48-diUb complex using NMR spectroscopy. a-c CSP averaged over ${ }^{1} \mathrm{H}$ and ${ }^{15} \mathrm{~N}$ dimensions of backbone amide protons upon the formation of the complex with $0.2 \mathrm{mM}$ isotope-labeled and unlabeled proteins. Insets: the ${ }^{15} \mathrm{~N}$-labeled subunit is illustrated with surface representation, and the red colored residues have CSPs above the dotted line. $\mathbf{d}$, e With a paramagnetic probe conjugated at E24C site of the distal Ub, the intensity ratios between paramagnetic and diamagnetic spectra, and the PRE $\Gamma_{2}$ values were assessed for backbone amide protons of ${ }^{15} \mathrm{~N}$-labeled Rpn $13^{\mathrm{NTD}}$. Gray boxes indicated residues with very large intermolecular PREs. $\mathbf{f}$ With the paramagnetic probe

conjugated at $\mathrm{N} 25 \mathrm{C}$ site of the proximal Ub, $\Gamma_{2}$ values were measured for amide protons of ${ }^{15} \mathrm{~N}$-labeled distal Ub in K48-diUb. The error bars indicate 1 S.D., and residues completely broadened out in the complex are denoted with stars 


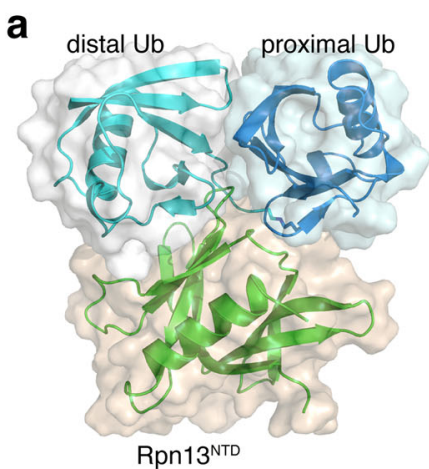

C

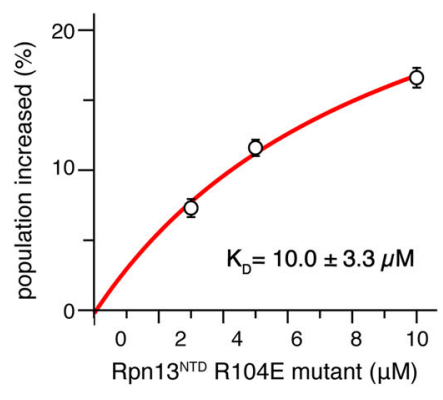

b
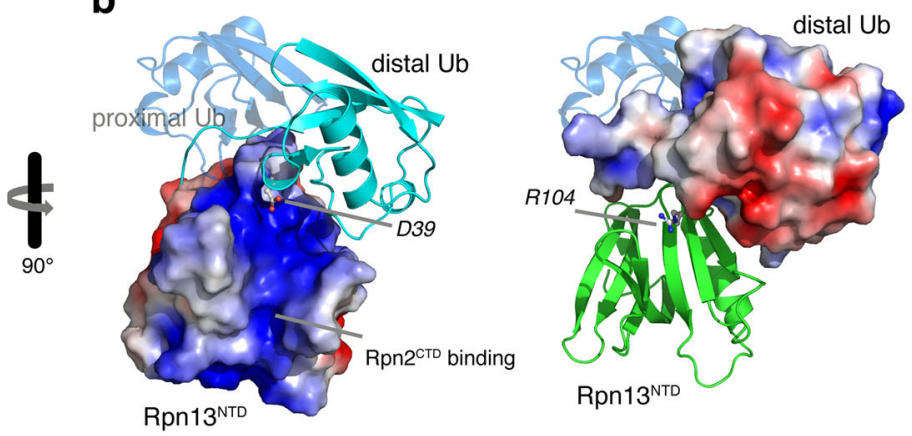

e

d

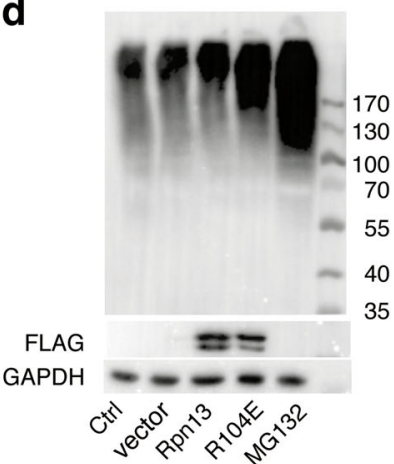

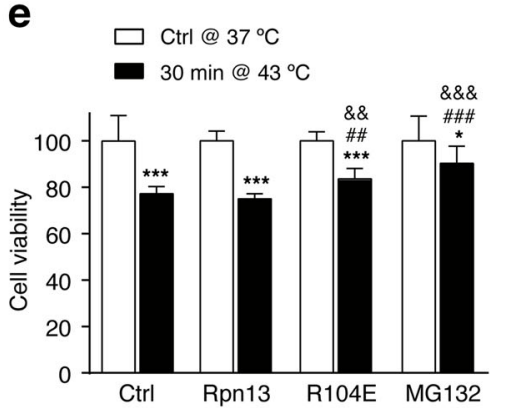

Fig. 4 Biological significance for the interaction between Rpn $13^{\mathrm{NTD}}$ and the distal Ub of K48-diUb. a Structure of Rpn13 $3^{\mathrm{NTD}}: \mathrm{K} 48$-diUb complex. Formation of the complex buries extensive interfaces between the subunits. $\mathbf{b}$ Electrostatic potential surfaces of Rpn $13^{\mathrm{NTD}}$ and distal Ub of K48-diUb, drawn at $\pm 3 k_{B}$ T scale. Residues R104 in Rpn13 ${ }^{\text {NTD }}$ and D39 in distal Ub are shown as balls-and-sticks. c R104E charge-reversal mutation in Rpn13 ${ }^{\text {NTD }}$ causes a 300-fold decrease in the binding affinity for K48-diUb, as assessed by smFRET (cf. Supplementary Fig. S11). The $K_{D}$ value is reported as best fit \pm fitting errors. $\mathbf{d}$ Western blot analyses show that the transfection of Rpn13 R104E mutant (with an N-terminal flag) increases the overall amount of K48-linked polyUb proteins in cell. e Cell viabilities upon heat shock (relative to cells without heat shock) show that transfection of Rpn13 R104E mutant, but not of wildtype Rpn13, confers thermotolerance. ${ }^{*} P<0.05$, ${ }^{* * *} P<0.001$, to control cells in the same group, unpaired $t$-test; ${ }^{\# \#} P<0.01$, ${ }^{\# \# \#} P$ $<0.001$, to the untreated cells with heat shock, one-way ANOVA; ${ }^{\&} P<<0.01$, \&\&\& $P<0.001$, to wildtype Rpn 13 transfected cells with heat shock, oneway ANOVA

Fig. S10a). Interestingly, though the hydrophobic residues L8, I44 and V70 in the proximal Ub are involved for interacting with Rpn13, the same three residues in the distal $\mathrm{Ub}$ are buried in Ub-Ub interface.

The Rpn13 ${ }^{\mathrm{NTD}}:$ K48-diUb complex structure can also be corroborated by single-molecule FRET data. Based on the complex structure, we modeled the fluorophores at their conjugation sites in K48-diUb. The average distance is $43.2 \pm 5.8 \AA$ between the geometric centers of fluorophore aromatic rings, which corresponds to a theoretical FRET efficiency of $0.73 \pm 0.13$ (Supplementary Fig. S10b). This value is almost the same as the center efficiency observed for the high-FRET species (Fig. 1a).

\section{Disruption of Rpn13 ${ }^{\mathrm{NTD}}$ : distal Ub interaction causes accumulation of ubiquitinated proteins in cell}

In the complex structure between Rpn $13^{\mathrm{NTD}}$ and K48diUb, the interaction between Rpn $13^{\mathrm{NTD}}$ and the proximal $\mathrm{Ub}$ is similar to that between $\mathrm{Rpn} 13^{\mathrm{NTD}}$ and $\mathrm{Ub}$ monomer, as previously reported (Supplementary
Fig. S10a). Thus, we designed experiments to assess functional importance for the interaction between Rpn $13^{\mathrm{NTD}}$ and the distal Ub of K48-diUb. Many charged residues are located at the interface between Rpn13 $3^{\text {NTD }}$ and the distal $\mathrm{Ub}$ of $\mathrm{K} 48-\mathrm{diUb}$, and therefore electrostatic force may play an important role for stabilizing the complex (Fig. 4b). Among them, residue D39 in the distal Ub is close to residue R104 in Rpn13. We thus mutated Rpn13 residue R104 to a glutamate, and titrated the mutant Rpn13 ${ }^{\text {NTD }}$ to fluorophore-labeled K48-diUb. The mutant protein enriches the high-FRET species of K48diUb (Supplementary Fig. S11). However, the binding affinity becomes much weaker. The binding isotherm can be fitted to a $K_{D}$ value of $10.0 \pm 3.3 \mu \mathrm{M}$, about 300 times weaker than the wildtype Rpn13 ${ }^{\text {NTD }}$ (Fig. 4c). As such, the association with the distal Ub is important for the specific recognition between Rpn13 and K48-diUb.

The decreased binding affinity of the R104E mutant allowed us to assess the functional importance of the interaction between Rpn13 and K48-linked Ub chain. 
a

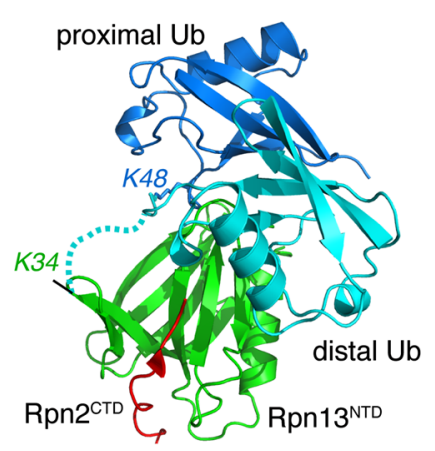

b

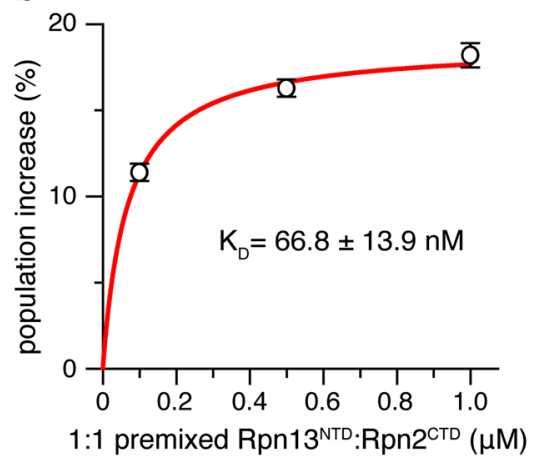

C

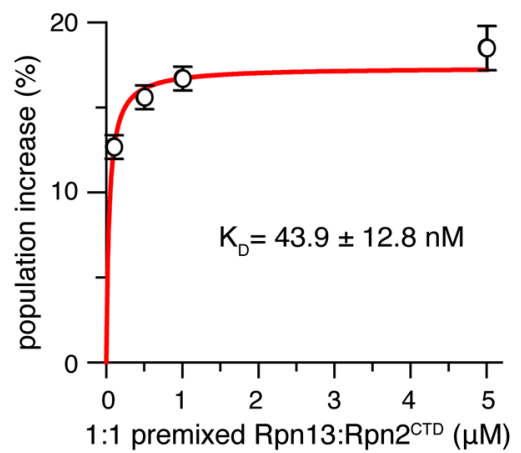

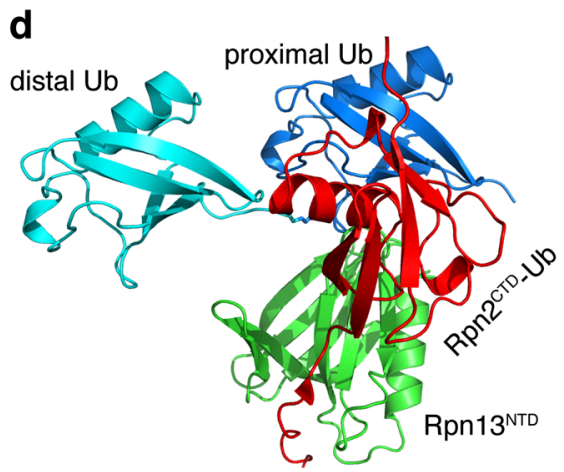

e $\quad+200 \mathrm{nM} \operatorname{Rpn}_{13}^{\mathrm{NTD}},+150 \mathrm{pM} \mathrm{Rpn2}^{\mathrm{CTD}-\mathrm{Ub}}$

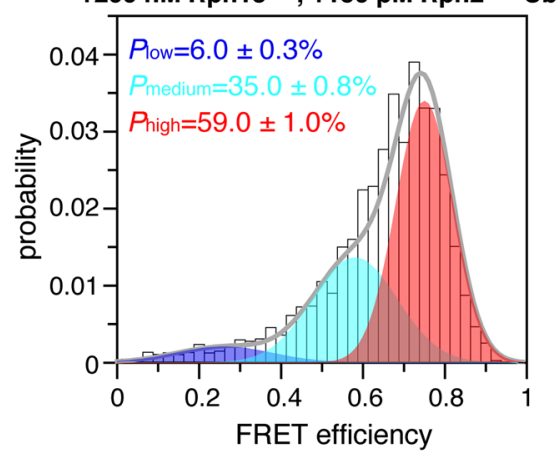

$\mathbf{f}$

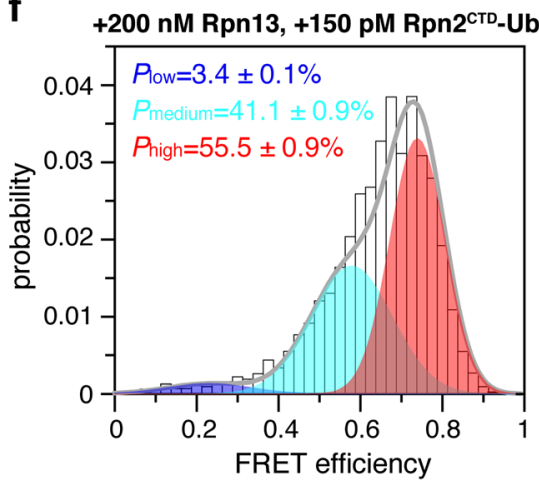

Fig. 5 Ub binding surface on Rpn13 $3^{\text {NTD }}$ can be targeted. a The binding surface of the distal Ub of K48-diUb on Rpn1 $3^{\text {NTD }}$ is adjacent to the binding surface of Rpn2 $2^{C T D}$. With Rpn13 $3^{N T D}-R p n 2^{C T D}$ complex structure (PDB code $5 \mathrm{~V} 1 \mathrm{Y}$ ) superimposed by Rpn13 $3^{\mathrm{NTD}}, \mathrm{Rpn}^{\mathrm{CTD}}$ is shown as red cartoon. Rpn13 residue K34 is close to the C-terminus of distal Ub (shown as a dotted line). b, c Binding affinities were obtained from smFRET titrations of equimolar Rpn13 ${ }^{\text {NTD }}$ :Rpn2 ${ }^{\text {CTD }}$ or Rpn13:Rpn2 ${ }^{\text {CTD }}$ to fluorophore-labeled K48-diUb. The $K_{D}$ values are reported as best fit \pm fitting errors. $\mathbf{d}$ Binding of Rpn2-anchored Ub monomer likely occupies the same binding surface of the distal Ub, causing the displacement of the latter. $\mathbf{e}$, $\mathbf{f}$ The smFRET competition experiments, with further addition of $150 \mathrm{pM} \mathrm{Rpn} 2^{\mathrm{CTD}}-\mathrm{Ub}$ fusion protein, to the mixture of $200 \mathrm{nM}$ Rpn $13^{\mathrm{NTD}}$ or full-length Rpn13 and 150 pM fluorophore-labeled K48-diUb (c.f. Figure 1C and f). The error indicates 1 S.D. from three independent measurements of the populations of the smFRET species

Transient transfection of wildtype Rpn13 slightly increases the amount of K48-linked polyUb proteins (Fig. 4d). It is possible that, upon Rpn13 transfection, an excess amount of free Rpn13 compete for binding to the K48linked polyUb proteins with proteasome-associated Rpn13, thus making the recruitment of ubiquitinated substrate proteins to the proteasome less efficient. On the other hand, the transfection of Rpn13 R104E mutant greatly increases the amount of K48-linked polyUb proteins, as compared to the cells transfected with wildtype Rpn13 (Fig. 4d). As a positive control, we incubated the cells with $1 \mu \mathrm{M}$ MG132, a potent proteasome inhibitor ${ }^{36}$. Due to the blockage of degradation of labile proteins, addition of MG132 significantly increases the amount of K48-linked polyUb proteins. Taken together, R104E mutation of Rpn13 can lead to the accumulation of 
ubiquitinated substrate proteins. This can be attributed to weaker interaction between proteasome-associated Rpn13 mutant and K48-diUb and K48-poyUb.

Heat shock can decrease cell viability. We found that 30 min heat shock at $43{ }^{\circ} \mathrm{C}$ can decrease the viability of HEK293 cells to $75 \%$. Similar to the previous reports ${ }^{36,37}$, we also found that the treatment of MG132 has a protective effect on cell survival upon heat shock, with cell viability decreased to $\sim 90 \%$ (Fig. 4e). This is because MG132 inhibits proteasomal degradation, making the otherwise short-living heat shock proteins more available (Fig. 4d). We also assayed the viability of heat-shocked cells transfected with wildtype Rpn13, and found no significant difference from the control cells without Rpn13 transfection. On the other hand, cell viability of Rpn13 R104E transfected cells decreased to $~ 83 \%$ upon heat shock, which is significantly higher than that of control cells and cells transfected with wildtype Rpn13 (Fig. 4e). This implies that the mutant Rpn13 has a protective effect on cell survival upon heat shock, similar to the effect of MG132. Taken together, the interaction between Rpn13 and K48-linked Ub chain is essential for Rpn13-mediated recognition of ubiquitinated substrate proteins by the proteasome, while an interfacial point mutation in Rpn13 can cause accumulation of certain substrate proteins such as heat shock proteins, and confer thermotolerance to the transfected cells.

\section{Rpn13 ${ }^{\mathrm{NTD}}:$ K48-diUb interaction can be targeted to modulate Rpn13 function}

Rpn13 is dynamically recruited to the proteasome via the interaction between Rpn $13^{\mathrm{NTD}}$ and the C-terminal tail of $\mathrm{Rpn} 2^{7,13}$. The complex structure here indicates that the binding interface on Rpn13 ${ }^{\mathrm{NTD}}$ for Rpn2 is close to but does not overlap with the binding interface for the distal Ub of K48-diUb (Fig. 5a). We thus premixed the last 16 residues of Rpn2 (Rpn2 ${ }^{\mathrm{CTD}}$ ) with Rpn13 ${ }^{\mathrm{NTD}}$ or with fulllength Rpn13 at 1:1 ratio, and titrated Rpn $13^{\mathrm{NTD}}: \operatorname{Rpn} 2^{\mathrm{CTD}}$ complex to fluorophore-labeled K48-diUb. The premixing of Rpn2 ${ }^{\text {CTD }}$ increased the $K_{D}$ value of $R p n 13^{\text {NTD }}: K 48$ diUb from $33.1 \pm 6.9 \mathrm{nM}$ (Figs. $1 \mathrm{~g}$ ) to $66.8 \pm 13.9 \mathrm{nM}$ (Supplementary Fig. S12a-c and Fig. 5b), while decreased the $K_{\mathrm{D}}$ value of Rpn13:K48-diUb from $119 \pm 24 \mathrm{nM}$ (Figs. 1d) to $43.9 \pm 12.8 \mathrm{nM}$ (Supplementary Fig. S12d-f and Fig. $5 \mathrm{c}$ ). Thus, providing the measurement uncertainties, the association of Rpn2 ${ }^{\mathrm{CTD}}$ cause only small perturbation for the binding affinity between Rpn13 and K48-diUb, which may also have to do with the presence of linker and the C-terminal domain of Rpn13.

Rpn2 and distal Ub of K48-diUb occupy nearby surfaces on $\mathrm{Rpn} 13^{\mathrm{NTD}}$. Therefore, a fusion protein with $\mathrm{Ub}$ monomer appended at the C-terminus of Rpn2 ${ }^{\mathrm{CTD}}$ may protrude out and interfere with the interaction between Rpn $13^{\text {NTD }}$ with the distal Ub of K48-diUb (Fig. 5d). As we have shown, addition of $200 \mathrm{nM}$ Rpn $13^{\mathrm{NTD}}$ increases the population of the high-FRET species of $150 \mathrm{pM}$ fluorophore-labeled K48-diUb to $\sim 63 \%$ (Fig. 1f), while addition of Ub monomer cannot compete for Rpn13NTD binding (Fig. 2c, d). When we added additional $150 \mathrm{pM}$ unlabeled Rpn2 ${ }^{\text {CTD }}$-Ub fusion protein, the population of high-FRET species decreases by $\sim 4 \%$ to $~ 59 \%$ (Fig. 5e). On the other hand, we have shown that, addition of $200 \mathrm{nM}$ full-length Rpn13 increases the population of the high-FRET species of $150 \mathrm{pM}$ fluorophore-labeled K48diUb to $\sim 60 \%$ (Fig. 1c). Further addition of $150 \mathrm{pM}$ unlabeled Rpn2 ${ }^{\text {CTD }}$-Ub fusion protein decreases the population of high-FRET species by $\sim 4.5$ to $\sim 55.5 \%$ (Fig. 5f). Note that appending a Ub at Rpn $2^{\mathrm{CTD}}$ has almost no effect on the interaction between $\mathrm{Rpn} 2^{\mathrm{CTD}}$ and Rpn13 ${ }^{\text {NTD }}$ (Supplementary Fig. S13). Thus, our data indicate that Rpn2 and K48-diUb binding interfaces on Rpn $13^{\text {NTD }}$ are close to each other. More importantly, Rpn2-anchored Ub can physically block the access of the distal diUb to Rpn13, and weaken the interaction between K48-diUb and Rpn13.

\section{Discussion}

In the present study, we have characterized the conformational dynamics of K48-diUb, and elucidated how Rpn13, a proteasomal receptor for ubiquitinated substrate proteins, can specifically interact with K48-linked Ub chain. Our finding thus explains linkage specificity of Rpn13-Ub interaction, and fills a missing piece of the puzzle about proteasomal receptor recognition-specific interactions with Rpn10, Rpn1 and Rpn13 all require the presence of at least two covalently linked Ub subunits. Using the smFRET technique, we show that K48-diUb fluctuates among three conformational states, and the high-FRET species is selectively enriched by $R p n 13^{\text {NTD }}$. The selectivity also holds for full-length Rpn13 and for K48-trtraUb. The smFRET titrations further indicate that Ub monomer, K63-linked diUb, and M1-linked diUb do not interact with Rpn13. Thus, K48-diUb and K48-polyUb are preferred binding partner of Rpn13.

Using NMR spectroscopy, we have determined the solution structure of the complex between Rpn $13^{\mathrm{NTD}}$ and K48-diUb by refining against intermolecular NOE and PRE restraints. The complex structure shows that the interaction between the distal Ub and Rpn $13^{\mathrm{NTD}}$ is mainly electrostatic and possibly dynamic, which can explain the sparsity of inter-subunit NOEs between the distal Ub and Rpn $13^{\mathrm{NTD}}$. Providing that the distal Ub of K48-diUb transiently opens up, noncovalent interactions can occur between Ub subunits ${ }^{22}$. This would explain a 2:1 stoichiometry between K48-diUb and Rpn $13^{\mathrm{NTD}}$, or a 1:1 stoichiometry between Ub monomer and Rnpn $13^{\text {NTD }}$ previously observed in bulk titrations ${ }^{3,7,9}$. Indeed, the Rpn $13^{\text {NTD }}$-Ub complex has been crystalized as a dimer 
(Supplementary Fig. S14) ${ }^{7}$. Herein, the structure of Rpn13 ${ }^{\mathrm{NTD}}:$ K48-diUb complex clearly explains how the two Ub subunits of $\mathrm{K} 48$-diUb simultaneously interact with Rpn13 ${ }^{\mathrm{NTD}}$. The NMR structure is also corroborated by the smFRET data, as the calculated and observed FRET efficiencies agree with each other (Supplementary Fig. S9b).

We have also shown that Rpn13 ${ }^{\mathrm{NTD}}$ :K48-diUb interaction can be disrupted by a charge-reversal mutation introduced at Rpn $13^{\mathrm{NTD}}$ facing the distal Ub of K48-diUb, or by blocking the access of the distal $\mathrm{Ub}$ with an $\mathrm{Ub}$ monomer fused to Rpn $2^{\mathrm{CTD}}$ and anchored at adjacent binding site. Rpn13 itself can be ubiquitinated at residue $\mathrm{K}^{2} 4^{21}$. In Rpn13 ${ }^{\mathrm{NTD}}:$ K48-diUb complex structure, Rpn13 residue $\mathrm{K} 34$ is much closer to the $\mathrm{C}$-terminus of the distal $\mathrm{Ub}$ of K48-diUb than to the C-terminus of the proximal Ub (Fig. 5a). Owing to high effective concentration, a Ub covalently attached at Rpn13 residue K34 can compete with the distal Ub of K48-diUb for Rpn13 binding, but not with the proximal $\mathrm{Ub}$ or $\mathrm{Ub}$ monomer as previously proposed $^{21}$. Thus, the present study explains how autoubiquitination can inhibit the function of Rpn13. The binding surface of the distal Ub on Rpn $13^{\text {NTD }}$ can also be a novel target of pharmaceutical intervention of proteasomal function.

In summary, we have used smFRET to analyze the number of inter-converting conformational states and their respective populations, and used NMR spectroscopy to determine the structure of the constituting conformational state. Both experiments were conducted in solution, and the FRET data measured for individual molecule and NMR data measured for bulk molecules corroborate each other. As such, the conjoint use of bulk and singlemolecule measurements gives us better insight of protein dynamics and the mechanism of protein function. We envision such integrative use of the two solution-based techniques can be generally used for characterizing other multi-subunit and multi-domain proteins.

\section{Materials and methods}

\section{Protein sample preparation}

Human ubiquitin was cloned to pET-11a vector (Novagen), and point mutations were introduced using the QuikChange strategy using Kod plus enzyme (Toyobo). To ensure a single final product of diubiquitin, K48R (or K63R) mutation was introduced to the distal Ub and 77D was introduced to the proximal Ub. For sitespecific conjugation of fluorescent probes (or paramagnetic probe), cysteine mutations were included to ubiquitin, one at a time, including $76 \mathrm{C}$ (mutated from Gly76), 25 C (mutated from Asn25), 24 C (mutated from Glu24), and $0 \mathrm{C}$ (MSAC sequence appended at the Nterminus). Human Rpn13 ${ }^{\text {NTD }}$ (residues 1-150) was cloned into a pET-11a vector. With a C91A mutation introduced to human OTUB1 (residues 40-271) and with a hexa-histidine tag appended at protein $\mathrm{N}$-terminus, the resulting protein, OTUB1i, has no deubiquitinase activity and was also cloned to pET-11a vector. Rpn2 ${ }^{\mathrm{CTD}}$ (GPQEPEPPEPFEYIDD in sequence at the very Cterminus of Rpn2) was purchased from GL Biochem (Shanghai). Rpn2 ${ }^{\text {CTD }}$ was also appended at the $\mathrm{N}$-terminus of ubiquitin.

Ubiquitin monomer, M1-diUb, or Rpn2 ${ }^{\mathrm{CTD}}$-Ub was expressed in BL21 star cells; the expression was induced with the addition of $1 \mathrm{mM}$ IPTG at $\mathrm{OD}_{600}$ value of $\sim 0.8$, and the culture was continued to grow at $37^{\circ} \mathrm{C}$ for $\sim 4 \mathrm{~h}$. Expression of $\mathrm{Rpn} 13^{\mathrm{NTD}}$ or full-length $\mathrm{Rpn} 13$ was induced with the addition of $0.2 \mathrm{mM}$ IPTG at $\mathrm{OD}_{600}$ of 0.8 , and the culture was gown overnight at $20^{\circ} \mathrm{C}$. Expression of OTUB1i was induced with the addition of $50 \mu \mathrm{M}$ IPTG at $\mathrm{OD}_{600}$ of 0.8 , and the culture was gown overnight at $16^{\circ} \mathrm{C}$. For expressing isotopically labeled protein, $\mathrm{U}_{-}{ }^{15} \mathrm{~N}$-labeled $\mathrm{NH}_{4} \mathrm{Cl}$ and $\mathrm{U}_{-}{ }^{13} \mathrm{C}$-labeled glucose (Sigma-Aldrich) were used as the sole nitrogen and carbon sources, and the cells were grown in M9-minimum medium prepared either $\mathrm{H}_{2} \mathrm{O}$ or $\mathrm{D}_{2} \mathrm{O}$ (Sigma-Aldrich). Rpn13 ${ }^{\mathrm{NTD}}$, ubiquitin (or ubiquitin mutants), or M1-diUb was purified on Sepharose SP, Sephacryl S100 and Source-S columns. OTUB1 was purified from His-affinity and Superdex-75 columns. The full-length Rpn13 was purified with sequential use His-Trap, Source-Q and Sephacryl S100 columns (GE Healthcare). Rpn2 ${ }^{\text {CTD }}$-Ub was purified using Sepharose Q, Source-Q and Sephacryl S100 sequentially. The identities of the proteins were confirmed using electrospray ionization mass spectrometry (Bruker Daltonics), and the protein concentrations were measured based on UV absorptions at $280 \mathrm{~nm}$.

The ligation between two ubiquitin proteins to afford a diubiquitin was performed following the established protocol $^{38}$. To ensure a unique ligation product, $0.5 \mathrm{mM}$ proximal $\mathrm{Ub}$ appended with residue D77 at the Cterminus was mixed equimolar with the distal Ub carrying a K48R mutation in $\mathrm{pH} 8.0$ buffer with $10 \mathrm{mM}$ Tris $\bullet \mathrm{HCl}$ and $1 \mathrm{mM}$ DTT. A mixture of $2.5 \mu \mathrm{M} \mathrm{E} 1,20 \mu \mathrm{M}$ E2-25K, $2 \mathrm{mM}$ ATP, $5 \mathrm{mM} \mathrm{MgCl}, 10 \mathrm{mM}$ creatine phosphate (Sigma-Aldrich, catalogue number 27920), $2 \mathrm{U} / \mathrm{ml}$ inorganic pyrophosphatase (Sigma-Aldrich, catalogue number I1643), and $1 \mathrm{U} / \mathrm{ml}$ creatine phosphokinase (SigmaAldrich, catalogue number C3755) were added for the reaction. The ligation took place at $30^{\circ} \mathrm{C}$ for $6 \mathrm{~h}$ and was quenched with the addition of $5 \mathrm{mM}$ DTT and $5 \mathrm{mM}$ EDTA. The K48-diUb protein was purified from the reaction mixture using Superdex 75 and Source-S columns (GE Healthcare) in tandem. To prepare K48-diUb with site-specific cysteine mutations, mutant proteins were used as the starting material for enzyme-catalyzed ligation reaction. Two copy of human ubiquitin genes were fused together to generate M1-linked diubiquitin 
(M1-diUb). M1-diUb protein was prepared in the same way as Ub monomer. K63-linked diubiquitin (K63-diUb) was prepared as described previously ${ }^{23}$.

We used Alexa Fluor $488 \mathrm{C}_{5}$ maleimide (ThermoFisher, catalogue number A10254) as the fluorescence donor and Cy5 maleimide (GE Healthcare, catalogue number PA15131) as the fluorescence acceptor. The Förster distance $R_{0}$ between the dyes is $52 \AA^{39}$. The dyes were first dissolved in DMSO at $1 \mathrm{mM}$ concentration, and were freshly prepared just before conjugation. The DTT in the buffer for cysteine mutant of K48-diUb was removed by desalting (HiPrep 26/10 Desalting column, GE Healthcare), and the protein was reacted with the premixed excess Alexa488 and Cy5 dyes. The conjugation reaction was performed in dark at room temperature for $4 \mathrm{~h}$, and the product was purified from the reaction mixture using Source-Q column (GE Healthcare). The doubly labeled K48-diUb had absorptions at 280, 493 and $650 \mathrm{~nm}$ all three wavelengths, and was further confirmed using mass spectrometry (Bruker Daltonics). Similarly, mixed labeling of fluorophores were introduced at $76 \mathrm{C}$ site of the proximal $\mathrm{Ub}$ and $25 \mathrm{C}$ site of the distal $\mathrm{Ub}$ in K63-diUb and M1-diUb.

To prepare K48-linked tetra-ubiquitin (K48-tetraUb), residue $\mathrm{D} 77$ of the proximal $\mathrm{Ub}$ in $\mathrm{K} 48$-diUb was deblocked using yeast ubiquitin C-terminal hydrolase-1 (YUH1), which was then ligated to another Ub77D protein, thus to generate K48-linked tri-ubiquitin (K48triUb). Subsequently, residue D77 in the most proximal $\mathrm{Ub}$ of K48-triUb was deblocked and was ligated to a fourth Ub77D protein to eventually generate K48-tetraUb. To prepare K48-tetraUb with cysteine mutations at specific sites, the mutant proteins were used as the starting material for enzyme-catalyzed ligation reactions. Fluorophore conjugation was performed as described above for K48-diUb.

\section{Single-molecule FRET data collection and analysis}

A Nikon A1 confocal microscope was used for singlemolecule imaging, and a pulsed interleaved excitation (PIE) scheme ${ }^{40}$ was employed, by using two SPCM$\mathrm{AQRH}$ detectors (Excelitas) to record the fluorescence time traces at the emission wavelengths of donor and acceptor. Two picosecond pulsed diode laser heads (LDHP-C-485B and LDH-P-C-640B, PicoQuant) were driven by a PDL 828 Sepia II driver (PicoQuant) at $32 \mathrm{MHz}$ repetition rate and were used for the interleaved excitations of Alexa488 and Cy5. Each laser was coupled to the A1 microscope through a single-mode fiber and was reflected by a dichroic mirror through a water immersion objective (WI $60 \times$, NA 1.20, Nikon).

The protein sample was loaded to a hybridization chamber (Thermo Fisher, catalogue number S24732). The fluorescence outputs were recorded with TimeHarp 260
PCI board (PicoQuant) built into a PC workstation, and the data were stored in the time-tagged time-resolved module (TTTR, PicoQuant). The photon counts for donor and acceptor emissions were analyzed by bursts ${ }^{41,42}$ using the parameters previously described ${ }^{43}$.

The smFRET measurements were performed at $25^{\circ} \mathrm{C}$ in $20 \mathrm{mM} \mathrm{pH}$ 7.4 HEPES buffer containing $150 \mathrm{mM} \mathrm{NaCl}$, $0.01 \%$ (v/v) Tween 20 (Thermo Fisher), $1 \mathrm{mM}$ ascorbic acid and $1 \mathrm{mM}$ methylviologen (Sigma-Aldrich). The concentration of the doubly labeled K48-diUb was $\sim 150$ pM. All other unlabeled protein samples were all prepared as stock solutions in $20 \mathrm{mM} \mathrm{pH}$ 7.4 HEPES buffer containing $150 \mathrm{mM} \mathrm{NaCl}$ and $4 \mathrm{mM}$ DTT and were mixed with K48-diUb prior to each experiment to the final desired concentration. The time traces were binned with 1 -ms bins and the threshold for photon count traces was typically 3-5 counts/bin per burst depending on the background dark counts. With high concentrations of ubiquitin receptors added, the threshold for photon count could reach 10 counts/bin, owing to higher background fluorescence.

To determine the number of constituting conformational states for K48-diUb from the smFRET data, we used expectation maximization (EM) algorithm ${ }^{32}$ for fitting multi-Gaussian mixture model using an in-house program. First, the program makes the hypothesis about the number of different components that make up the observed smFRET data, and then it calculates the likelihood for the corresponding parameters, and finally the program adjusts the parameters to maximize the likelihood. For evaluating the number of Gaussian components, we introduced Bayesian information criterion (BIC) and Akaike information criterion (AIC) ${ }^{44,45}$, in addition to the correlation coefficient $R^{2}$. The smFRET measurement and fitting were repeated three times, and the averaged FRET efficiencies, populations and associated standard deviations were reported. To estimate the $K_{\mathrm{D}}$ value, the populational increase of a particular smFRET species was fitted against the concentration of unlabeled protein added in Origin 8.0, using the following equation:

$$
\begin{aligned}
& \Delta P_{\text {FRET }}= \\
& \frac{P_{\text {max }}\left(C_{\text {partner }}+C_{\text {diub }}+K_{\mathrm{D}}-\sqrt{\left(C_{\text {Partner }}+C_{\text {diUb }}+K_{\mathrm{D}}\right)^{2}-4 C_{\text {partner }} C_{\text {diUb }}}\right)}{2 C_{\text {diub }}} .
\end{aligned}
$$

Here $\Delta P_{\text {FRET }}$ is the change of the population of a particular FRET species, $P_{\max }$ is the maximum difference for the FRET species with or without the partner protein, $C_{\text {partner }}$ is the concentration of the partner protein added, $C_{\mathrm{diUb}}$ is the concentration of the fluorophore-conjugated K48-diUb. 


\section{NMR data acquisition and analysis}

For the assignment of NMR resonances of Rpn $13^{\text {NTD }}$, $\mathrm{U}-\left[{ }^{2} \mathrm{H},{ }^{13} \mathrm{C},{ }^{15} \mathrm{~N}\right]$ - and $\mathrm{U}-\left[{ }^{13} \mathrm{C},{ }^{15} \mathrm{~N}\right]$-labeled proteins were prepared in $20 \mathrm{mMpH}$ 6.5 MES buffer containing $150 \mathrm{mM} \mathrm{NaCl}, 4 \mathrm{mM}$ DTT, and $10 \% \mathrm{D}_{2} \mathrm{O}$. The NMR data were collected for a $\sim 0.6 \mathrm{mM}$ sample at $25^{\circ} \mathrm{C}$ on a Bruker Avance III $850 \mathrm{MHz}$ spectrometer equipped with a TXI cryogenic probe. The ${ }^{1} \mathrm{H}_{-}{ }^{15} \mathrm{~N}$ HSQC, HNCA, HNCB and $\mathrm{HCCH}$-TOCSY experiments were used for backbone and side-chain resonance assignment; the final assignment was compared against the known assignment ${ }^{13}$.

To characterize the interactions between $\operatorname{Rpn} 13^{\mathrm{NTD}}$ and K48-diUb, K48-diUb with the proximal Ub ${ }^{15} \mathrm{~N}$-labeled was titrated with unlabeled Rpn13 ${ }^{\mathrm{NTD}}$, K48-diUb with the distal Ub ${ }^{15} \mathrm{~N}$-labeled was titrated with unlabeled Rpn $13^{\mathrm{NTD}}$, or ${ }^{15} \mathrm{~N}$-labeled Rpn $13^{\mathrm{NTD}}$ was titrated with unlabeled K48-diUb. Average CSP for each residue was calculated using the equation $\Delta \delta=\left[0.5\left(\Delta \delta_{\mathrm{H}}{ }^{2}+\right.\right.$ $\left.\left.0.2 \Delta \delta_{\mathrm{N}}^{2}\right)\right]^{1 / 2}$, in which $\Delta \delta_{\mathrm{H}}$ and $\Delta \delta_{\mathrm{N}}$ are the CSP values in ${ }^{1} \mathrm{H}$ and ${ }^{15} \mathrm{~N}$ dimensions. Both K48-diUb and Rpn $13^{\mathrm{NTD}}$ are $0.2 \mathrm{mM}$ in concentration, and the $1: 1$ complex contains ${ }^{15} \mathrm{~N}$-labeled and unlabeled proteins.

To obtain intermolecular NOEs, we prepared two NMR samples. In one sample, the distal Ub is Ub $\mathrm{U}-\left[{ }^{13} \mathrm{C},{ }^{15} \mathrm{~N}\right]-$ labeled, and the proximal $\mathrm{Ub}$ and $\mathrm{Rpn} 13^{\mathrm{NTD}}$ are unlabeled. In the other sample, Rpn $13^{\text {NTD }}$ is $\mathrm{U}-\left[{ }^{13} \mathrm{C},{ }^{15} \mathrm{~N}\right]-$ labeled, and $\mathrm{K} 48-\mathrm{diUb}$ is unlabeled. The concentrations were $0.5 \mathrm{mM}$ for the isotopically labeled protein, and $0.6 \mathrm{mM}$ for the unlabeled protein. Half-filtered ${ }^{13} \mathrm{C}$ NOESY experiments ${ }^{46}$ were performed with a $120 \mathrm{~ms}$ NOE mixing time. Intermolecular NOE cross-peaks were assigned manually and iteratively based on the initial structures for the ternary complex.

Cysteine point mutation was introduced either at the proximal Ub (N25C site) or distal Ub (E24C site), and the mutant protein with natural isotope abundance was conjugated to maleimide-EDTA- $\mathrm{Mn}^{2+}$ paramagnetic probe as previously described ${ }^{22,23}$. The conjugated product was further purified with Source-Q column, and was confirmed by mass spectrometry. The paramagnetically tagged $\mathrm{Ub}$ is enzymatically ligated to another $\mathrm{Ub}$ with either ${ }^{15} \mathrm{~N}$ enrichment (N25C site) or with natural isotope abundance (E24C site). Transverse relaxation rates $\Gamma_{2}$ were measured for the backbone amide protons of 0.3 $\mathrm{mM}$ K48-diUb using the established protocol ${ }^{47}$ in the absence or presence of equimolar Rpn13 ${ }^{\mathrm{NTD}}$.

\section{Structure calculation of the Rpn13 ${ }^{\mathrm{NTD}}: \mathrm{K} 48$-diUb complex}

Structural calculation was performed using Xplor$\mathrm{NIH}^{48}$ with conjoined torsion angle/rigid body simulated annealing refinement ${ }^{49}$. The starting structures of ubiquitin and Rpn $13^{\text {NTD }}$ were taken from PDB structures $1 \mathrm{UBQ}^{50}$ and $2 \mathrm{R} 2 \mathrm{Y}^{6}$, respectively. The initial structure of Rpn $13^{\mathrm{NTD}}$ was further refined using intramolecular NOE and residual dipolar coupling restraints collected in pf1 and PEG-hexanol media. The coordinates for ubiquitin were duplicated, and an isopeptide bond was patched between the proximal Ub and distal Ub. The refinement was performed with a target function comprising the intermolecular NOE restraints (between proximal Ub and distal Ub, between proximal $\mathrm{Ub}$ and $\mathrm{Rpn} 13^{\mathrm{NTD}}$, and between distal $\mathrm{Ub}$ and $\mathrm{Rpn} 13^{\mathrm{NTD}}$ ), a van der Waals repulsive term, and a radius-of-gyration restraint ${ }^{51}$ for the interfacial residues mapped by the NMR chemical shift perturbation, and the PRE-restraints with the paramagnetic probe patched at $\mathrm{N} 25 \mathrm{C}$ site of proximal $\mathrm{Ub}$ and at E24C of distal Ub (applied as squared-well potential with $\pm 10 \mathrm{~s}^{-1}$ upper/lower bounds).

To obtain an initial pose for the ternary complex, for residues $1-71$ of the proximal $\mathrm{Ub}$, residues $1-71$ of the distal Ub and Rpn $13^{\mathrm{NTD}}$, each was grouped as a rigid body, while complete torsion angle freedom was given to the isopeptide linker including the side chain of K48 in the proximal $\mathrm{Ub}$ and residues $72-76$ in the distal $\mathrm{Ub}$. Besides experimental restraints, the diubiquitin linker residues were subjected to torsion angle database restraint ${ }^{52}$ for better packing at the interfaces. The solvent accessible surface area (SASA) was calculated with Xplor$\mathrm{NIH}$ with a probe radius of $1.4 \AA$, and was subtracted from the SASA calculated from the sub of surface area of the free proteins to obtain the buried SASA. Based on the complex structure, fluorescent probes (maleimide AlexaFluor-488 and Cy5) were patched onto the labeling sites, and the linker between protein backbone and rigid portion of the fluorophore (also including residues 72-76 when labeled at the Ub C-terminus) are given torsion angle freedom and are allowed reorient. The distances between the fluorophores are calculated with $\left\langle r^{-6}\right\rangle$ averaging to afford expected FRET efficiencies. Structures were selected for their overall energy, and figures were illustrated using PyMol version 2.0 (Schrödinger LLC).

\section{Cell-based experiments}

Human Rpn13 cDNA was purchased (Sino Biologicals, Beijing, China), and was sub-cloned to a pcDNA3.1 vector. A flag tag was appended at the N-terminus of Rpn13, and R104E mutation was site-specifically introduced. The plasmids (pcDNA3.1, pcDNA3.1/flag-hRpn13, and pcDNA3.1/flag-hRpn13/R104E) were transfected to HEK293 cells (Cell Biology of the Chinese of Academy of Sciences, Shanghai, China) using PEI reagent (polyethyleneimine, m.w. 25,000, Alfa Aesar Chemical, Shanghai, China). As a positive control, $6 \mathrm{~h}$ after transfection, MG132 (at $1 \mu \mathrm{M}$ final concentration, SigmaAldrich, St. Louis MO, USA) was added to one group of untransfected cells. $48 \mathrm{~h}$ after transfection, the cells were washed twice with ice-cold phosphate-buffered saline (PBS) and then lysed at $4{ }^{\circ} \mathrm{C}$ in RIPA lysis buffer 
(Beyotime Biotechnology, Shanghai, China). Protein concentrations were determined by using $\mathrm{BCA}$ assay kit (Beyotime Biotechnology).

For western blotting, $50 \mu \mathrm{g}$ cell lysate was separated in 7.5\% SDS page gel, and transferred to $0.45 \mu \mathrm{m}$ PVDF membrane (Millipore Corporation, Billerica, USA). The membrane was blocked by $5 \%$ fat-free milk, and was incubated with rabbit anti-K48 linkage specific polyubiquitin antibody (Cell Signaling Technology, Boston, USA), rabbit anti-flag tag antibody (Proteintech, Wuhan, China) and mouse anti GAPDH antibody (Proteintech) at $4{ }^{\circ} \mathrm{C}$ overnight. After being washed three time by using TBST (Tris buffered saline with Tween 20, $50 \mathrm{mM}$ Tri$\mathrm{HCl}, 0.15 \mathrm{M}$ sodium chloride, $0.05 \%$ Tween $20, \mathrm{pH} 7.6$ ), the membrane was incubated in horse HRP-conjugated anti-mouse IgG antibody (Cell Signaling Technology) or goat HRP-conjugated anti-rabbit IgG antibody (Cell Signaling Technology) at room temperature for $2 \mathrm{~h}$. After being washed three times with TBST, the membrane was incubated with ECL (Enhanced Chemiluminescence, BioRAD, Hercules, USA), and the immunoblots were analyzed by ChemiDocTM MP V3 (Bio-RAD).

As a parallel experiment, $6 \mathrm{~h}$ after transfection, the three groups of transfected cells and two groups of untransfected cells were cultured in two 96-well plates (with $1 \mu \mathrm{M}$ MG132 added to one group of un-transfected cells). $24 \mathrm{~h}$ later, the cells in one 96-well plate was switched to $43^{\circ} \mathrm{C}$ culture temperature using pre-heated culture medium, and were cultured at $43^{\circ} \mathrm{C}$ for $30 \mathrm{~min}$. The heat-shocked cells were reversed to $37^{\circ} \mathrm{C}$, and were cultured for another $24 \mathrm{~h}$. The cells in the other 96-well plate was cultured under normal conditions at $37^{\circ} \mathrm{C}$. MTT assay was used to detect the cell viability; cells were incubated with $0.5 \mathrm{mg} / \mathrm{ml}$ of 3-(4,5-cimethylthiazol-2-yl)2,5-diphenyl tetrazolium bromide (MTT, Sigma-Aldrich) for $2 \mathrm{~h}$ at $37^{\circ} \mathrm{C}$. After the removal of the MTT solution, $100 \mu \mathrm{l}$ DMSO were added. Following another 10-minute incubation, the absorbance was read at $570 \mathrm{~nm}$ in a plate reader (Elx800, Bi-TEK Instrument USA). Cell viabilities were normalized to those of $37^{\circ} \mathrm{C}$ control cells.

\section{Accession codes}

Atomic coordinates for complex structure between K48-diUb and Rpn13 N-terminal domain have been deposited with the PDB with accession code of 5YMY and the associated NMR data have been deposited at BMRB with the accession code of 36127 .

\section{Acknowledgements}

The work has been supported by the National Key R\&D Program of China (2018YFA0507700 to C.T., W.P.Z., Z.L. and Z.G, 2016 YFA0501200 to C.T., Z.G. and X.D., and 2017YFA0505400 to X.D.) and by the National Natural Science Foundation of China (91753132 and 31770799 to C.T., 81573400 to W.P.Z., 31500595 to Z.L., 31400735 to Z.G., and 31400644 to X.D.).

\section{Author details}

${ }^{1}$ CAS Key Laboratory of Magnetic Resonance in Biological Systems, State Key Laboratory of Magnetic Resonance and Atomic Molecular Physics, National Center for Magnetic Resonance at Wuhan, Wuhan Institute of Physics and Mathematics of the Chinese Academy of Sciences, Wuhan, Hubei Province 430071, China. ${ }^{2}$ National Key Laboratory of Crop Genetic Improvement, College of Life Science and Technology, Huazhong Agricultural University, Wuhan 430070, China. ${ }^{3}$ University of Chinese Academy of Sciences, Beijing 100049, China. ${ }^{4}$ Department of Pharmacology, Institute of Neuroscience, Key Laboratory of Medical Neurobiology of Ministry of Health of China, and Zhejiang Province Key Laboratory of Mental Disorder's Management, Zhejiang University School of Medicine, Hangzhou, Zhejiang Province 310027, China. ${ }^{5}$ Wuhan National Laboratory for Optoelectronics, Wuhan, Hubei Province 430074, China

\section{Author contributions}

Z.L., W.P.Z. and C.T. designed the experiments, Z.L., H.-W.Y. and J.Y. prepared the samples, Z.L., H.-W.Y. and K.L. performed smFRET experiments and analyses, J.Y. and Y.W. performed cell-based experiments, X.D. and Z.G. determined the complex structure, W.P.Z. and C.T. wrote the manuscript with support from all authors.

Conflict of interest

The authors declare that they have no conflict of interest.

\section{Publisher's note}

Springer Nature remains neutral with regard to jurisdictional claims in published maps and institutional affiliations.

Supplementary Information accompanies the paper at (https://doi.org/ 10.1038/s41421-019-0089-7).

Received: 30 November 2018 Revised: 15 February 2019 Accepted: 19 February 2019

Published online: 02 April 2019

\section{References}

1. Shi, Y. et al. Rpn 1 provides adjacent receptor sites for substrate binding and deubiquitination by the proteasome. Science 351, aad9421 (2016).

2. Deveraux, Q., Ustrell, V., Pickart, C. \& Rechsteiner, M. A 26-S protease subunit that binds ubiquitin conjugates. J. Biol. Chem. 269, 7059-7061 (1994).

3. Husnjak, K. et al. Proteasome subunit Rpn13 is a novel ubiquitin receptor. Nature 453, 481-488 (2008).

4. Hierpe, R. et al. UBQLN2 mediates autophagy-independent protein aggregate clearance by the proteasome. Cell 166, 935-949 (2016).

5. Samant, R. S., Livingston, C. M., Sontag, E. M. \& Frydman, J. Distinct proteostasis circuits cooperate in nuclear and cytoplasmic protein quality control. Nature 563, 407-411 (2018).

6. Schreiner, P. et al. Ubiquitin docking at the proteasome through a novel pleckstrin-homology domain interaction. Nature 453, 548-552 (2008).

7. VanderLinden, R. T., Hemmis, C. W., Yao, T., Robinson, H. \& Hill, C. P. Structure and energetics of pairwise interactions between proteasome subunits RPN2, RPN13, and ubiquitin clarify a substrate recruitment mechanism. J. Biol. Chem. 292, 9493-9504 (2017).

8. Zhang, N. et al. Structure of the s5a:k48-linked diubiquitin complex and its interactions with rpn13. Mol. Cell 35, 280-290 (2009).

9. Chen, X. et al. Structures of Rpn1 T1:Rad23 and hRpn13:hPLIC2 reveal distinct binding mechanisms between substrate receptors and shuttle factors of the proteasome. Structure 24, (1257-1270 (2016).

10. Huang, X., Luan, B., Wu, J. \& Shi, Y. An atomic structure of the human 265 proteasome. Nat. Struct. Mol. Biol. 23, 778-785 (2016).

11. Chen, S. et al. Structural basis for dynamic regulation of the human 265 proteasome. Proc. Natl Acad. Sci. USA 113, 12991-12996 (2016).

12. Berko, D. et al. Inherent asymmetry in the 265 proteasome is defined by the ubiquitin receptor RPN13. J. Biol. Chem. 289, 5609-5618 (2014).

13. $L u, X$. et al. Structure of the Rpn13-Rpn2 complex provides insights for Rpn13 and Uch37 as anticancer targets. Nat. Commun. 8, 15540 (2017). 
14. Wang, $X$. et al. Molecular details underlying dynamic structures and regulation of the human 26s proteasome. Mol. Cell. Proteom. 16, 840-854 (2017).

15. Pilarsky, C., Wenzig, M., Specht, T., Saeger, H. D. \& Grutzmann, R. Identification and validation of commonly overexpressed genes in solid tumors by comparison of microarray data. Neoplasia 6, 744-750 (2004).

16. Anchoori, R. K. et al. A bis-benzylidine piperidone targeting proteasome ubiquitin receptor RPN13/ADRM1 as a therapy for cancer. Cancer Cell. 24, 791-805 (2013).

17. Trader, D. J., Simanski, S. \& Kodadek, T. A reversible and highly selective inhibitor of the proteasomal ubiquitin receptor rpn13 is toxic to multiple myeloma cells. J. Am. Chem. Soc. 137, 6312-6319 (2015).

18. Mazumdar, T. et al. Regulation of NF-kappa B activity and inducible nitric oxide synthase by regulatory particle non-ATPase subunit 13 (Rpn13). Proc. Natl Acad. Sci. USA 107, 13854-13859 (2010).

19. Wagner, S. A. et al. A proteome-wide, quantitative survey of in vivo ubiquitylation sites reveals widespread regulatory roles. Mol. Cell. Proteom. 10, M111 013284 (2011).

20. Kim, W. et al. Systematic and quantitative assessment of the ubiquitinmodified proteome. Mol. Cell 44, 325-340 (2011).

21. Besche, H. C. et al. Autoubiquitination of the $26 \mathrm{~S}$ proteasome on Rpn13 regulates breakdown of ubiquitin conjugates. EMBO J. 33, 1159-1176 (2014).

22. Liu, Z. et al. Noncovalent dimerization of ubiquitin. Angew. Chem. Int. Ed. 51 469-472 (2012).

23. Liu, Z. et al. Lys63-linked ubiquitin chain adopts multiple conformational states for specific target recognition. elife 4, e05767 (2015).

24. Cook, W. J., Jeffrey, L. C., Carson, M., Chen, Z. \& Pickart, C. M. Structure of a diubiquitin conjugate and a model for interaction with ubiquitin conjugating enzyme (E2). J. Biol. Chem. 267, 16467-16471 (1992).

25. Hirano, T. et al. Conformational dynamics of wild-type Lys-48-linked diubiquitin in solution. J. Biol. Chem. 286, 37496-37502 (2011).

26. Ye, Y. et al. Ubiquitin chain conformation regulates recognition and activity of interacting proteins. Nature 492, 266-270 (2012).

27. Berlin, $\mathrm{K}$. et al. Recovering a representative conformational ensemble from underdetermined macromolecular structural data. J. Am. Chem. Soc. 135, 16595-16609 (2013).

28. Liu, S. et al. Promiscuous interactions of gp78 E3 ligase CUE domain with polyubiquitin chains. Structure 20, 2138-2150 (2012).

29. Schuler, B. Single-molecule FRET of protein structure and dynamics - a primer. J. Nanobiotechnology 11, S2 (2013).

30. Dimura, M. et al. Quantitative FRET studies and integrative modeling unravel the structure and dynamics of biomolecular systems. Curr. Opin. Struct. Biol. 40, 163-185 (2016).

31. Lerner, E. et al. Toward dynamic structural biology: Two decades of singlemolecule Forster resonance energy transfer. Science 359, eaan1133 (2018).

32. Burnham, K. P. Multimodel inference: understanding AIC and BIC in model selection. Soci. Meth. Res. 33, 261-304 (2004).

33. Wiener, R., Zhang, X., Wang, T. \& Wolberger, C. The mechanism of OTUB1mediated inhibition of ubiquitination. Nature 483, 618-622 (2012).
34. Satoh, T. et al. Crystal structure of cyclic Lys48-linked tetraubiquitin. Biochem. Biophys. Res. Commun. 400, 329-333 (2010).

35. Liu, Z., Gong, Z., Dong, X. \& Tang, C. Transient protein-protein interactions visualized by solution NMR. Biochim. Biophys. Acta, Proteins Proteom. 1864, 115-122 (2016)

36. Lee, D. H. \& Goldberg, A. L. Proteasome inhibitors cause induction of heat shock proteins and trehalose, which together confer thermotolerance in Saccharomyces cerevisiae. Mol. Cell. Biol. 18, 30-38 (1998).

37. Kim, H. J. et al. Systemic analysis of heat shock response induced by heat shock and a proteasome inhibitor MG132. PLOS ONE 6, e20252 (2011).

38. Pickart, C. M. \& Raasi, S. Controlled synthesis of polyubiquitin chains. Methods Enzymol. 399, 21-36 (2005).

39. Kalinin, S. et al. A toolkit and benchmark study for FRET-restrained highprecision structural modeling. Nat. Methods 9, 1218-1225 (2012).

40. Muller, B. K., Zaychikov, E., Brauchle, C. \& Lamb, D. C. Pulsed interleaved excitation. Biophys. J. 89, 3508-3522 (2005).

41. Lee, N. K. et al. Accurate FRET measurements within single diffusing biomolecules using alternating-laser excitation. Biophys. J. 88, 2939-2953 (2005).

42. Gopich, I. V. Accuracy of maximum likelihood estimates of a two-state model in single-molecule FRET. J. Chem. Phys. 142, 034110 (2015).

43. Dong, $X$. et al. Ubiquitin $\mathbf{S} 65$ phosphorylation engenders a $\mathrm{pH}$-sensitive conformational switch. Proc. Natl Acad. Sci. USA 114, 6770-6775 (2017).

44. Akaike, H. A new look at the statistical model identification. IEEE Trans. Autom. Control 19, 716-723 (1974).

45. Schwarz, G. Estimating the dimension of a model. Ann. Stat. 6, 461-464 (1978).

46. Zwahlen, C. et al. Methods for measurement of intermolecular NOEs by multinuclear NMR spectroscopy: application to a bacteriophage lambda Npeptide/boxB RNA complex. J. Am. Chem. Soc. 119, 6711-6721 (1997).

47. Iwahara, J., Tang, C. \& Clore, G. M. Practical aspects of $1 \mathrm{H}$ transverse paramagnetic relaxation enhancement measurements on macromolecules. J. Magn. Reson. 184, 185-195 (2007).

48. Schwieters, C. D., Kuszewski, J. J. \& Clore, G. M. Using Xplor-NIH for NMR molecular structure determination. Prog. Nucl. Magn. Reson. Spectrosc. 48, 47-62 (2006).

49. Clore, G. M. Accurate and rapid docking of protein-protein complexes on the basis of intermolecular nuclear overhauser enhancement data and dipolar couplings by rigid body minimization. Proc. Natl Acad. Sci. USA 97, 9021-9025 (2000).

50. Vijay-Kumar, S., Bugg, C. E. \& Cook, W. J. Structure of ubiquitin refined at 1.8 A resolution. J. Mol. Biol. 194, 531-544 (1987).

51. Tang, C. \& Clore, G. M. A simple and reliable approach to docking proteinprotein complexes from very sparse NOE-derived intermolecular distance restraints. J. Biomol. NMR 36, 37-44 (2006).

52. Bermejo, G. A., Clore, G. M. \& Schwieters, C. D. Smooth statistical torsion angle potential derived from a large conformational database via adaptive kernel density estimation improves the quality of NMR protein structures. Protein Sci. 21, 1824-1836 (2012). 